\title{
A Greek multicenter study comparing the clinical and immunologic phenotypes between adult and juvenile- onset lupus
}

\author{
P Nalbanti ${ }^{1}$ S Stefanidou ${ }^{2}$, S Alfantaki ${ }^{3}$, A Siamopoulou ${ }^{3}$, M Trachana $^{1 *}$, V Galanopoulou ${ }^{4}$, P Pratsidou-Gerts ${ }^{1}$, \\ E Farmaki ${ }^{1}$, F Papachristou' ${ }^{1}$ A Garyphallos ${ }^{2}$, F Kanakoudi-Tsakalidou ${ }^{1}$ \\ From 18th Pediatric Rheumatology European Society (PReS) Congress \\ Bruges, Belgium. 14-18 September 2011
}

\section{Background and Aim}

To compare the clinical and immunologic phenotypes between Juvenile and Adult onset SLE (jSLE, aSLE) in Greek patients at disease onset and 5 yrs thereafter due to paucity of relevant data from Mediterranean countries.

\section{Methods}

This retrospective study enrolled 66 jSLE and 97 aSLE patients, all having been attended in 4 Rheumatology Centers. Demographic data, as well as clinical and immunologic findings at diagnosis and 5 yrs thereafter were studied.

\section{Results}

At diagnosis the mean (SD) ages were 12.25(0.27) and 33.93(1.32) yrs, whereas the mean follow-up 6.63 (0.59) and $11.6(0.7)$ yrs, for jSLE and aSLE respectively. General features, hepatosplenomegaly, lymphadenopathy and haematology abnormalities were more frequent in jSLE patients $(\mathrm{p} 1=0.001, \mathrm{p} 2=0.025$ and $\mathrm{p} 3<0.0001$, respectively), whereas photosensitivity was commoner in those with $\operatorname{aSLE}(\mathrm{p}=0.047)$. The main difference was the higher mean number of organ/system involvement in the jSLE group $(p=0.0006)$. At the end of $5 \mathrm{yrs}$, the cumulative number of clinical manifestations was similar in both groups. Anti-dsDNAs, anti-cardiolipin, anti-Sm,
anti-URNP antibodies and low C3 and C4 were significantly commoner in jSLE $(\mathrm{p}<0.01)$.

\section{Conclusions}

Findings of this study are in line with previous publications regarding the need for early aggressive therapy in jSLE due to the graver presentation at onset as compared to aSLE. However, the incidence of nephritis or CNS or skin involvement was not higher compared to aSLE, as previously reported. Moreover, no significant differences in clinical or immunologic phenotypes were cumulatively found between the 2 groups during the first 5 yrs of disease course.

\section{Author details}

${ }^{1} 1$ st Dept of Pediatrics, Aristotle University, Pediatric Immunology and Rheumatology Referral Center, Thessaloniki. ${ }^{2} 4$ th Dept of Internal Medicine, Aristotle University, Thessaloniki. ${ }^{3}$ Dept of Child Health, University of loannina, loannina. ${ }^{4}$ Rheumatology Unit, N. Papageorgiou Hospital, Thessaloniki, Greece.

Published: 14 September 2011

doi:10.1186/1546-0096-9-S1-P245

Cite this article as: Nalbanti et al:: A Greek multicenter study comparing the clinical and immunologic phenotypes between adult and juvenileonset lupus. Pediatric Rheumatology 2011 9(Suppl 1):P245.

\footnotetext{
* Correspondence: mtrachan@auth.gr

'1st Dept of Pediatrics, Aristotle University, Pediatric Immunology and

Rheumatology Referral Center, Thessaloniki

Full list of author information is available at the end of the article
}

(c) 2011 Nalbanti et al; licensee BioMed Central Ltd. This is an open access article distributed under the terms of the Creative Commons 